\title{
Berger Type Deformed Sasaki Metric and Harmonicity on the Cotangent Bundle
}

\author{
Abderrahim Zagane* \\ (Dedicated to the memory of Prof. Dr. Aurel BEJANCU (1946 - 2020))
}

\begin{abstract}
In this paper, we introduce the Berger type deformed Sasaki metric on the cotangent bundle $T^{*} M$ over an anti-paraKähler manifold $(M, \varphi, g)$. We establish a necessary and sufficient conditions under which a covector field is harmonic with respect to the Berger type deformed Sasaki metric. We also construct some examples of harmonic vector fields. we also study the harmonicity of a map between a Riemannian manifold and a cotangent bundle of another Riemannian manifold and vice versa.
\end{abstract}

Keywords: Cotangent bundles, horizontal lift and vertical lift, Berger type deformed Sasaki metric, harmonic maps.

AMS Subject Classification (2020): Primary: 53A45; 53C43; 58E20; Secondary: 53C20; 53 C55.

\section{Introduction}

In the field, one of the first works which deal with the cotangent bundles of a manifold as a Riemannian manifold is that of Patterson, E. M., Walker, A. G. [9] who constructed from an affine symmetric connection on a manifold a Riemannian metric on the cotangent bundle, which they call the Riemann extension of the connection. A generalization of this metric had been given by Sekizawa, M.[12] in his classification of natural transformations of affine connections on manifolds to metrics on their cotangent bundles, obtaining the class of natural Riemann extensions which is a 2-parameter family of metrics, and which had been intensively studied by many authors. On the other hand, inspired by the concept of g-natural metrics on tangent bundles of Riemannian manifolds, A $\breve{g c a}$, F. considered another class of metrics on cotangent bundles of Riemannian manifolds, that he callad g-natural metrics [1]. Also, there are studies by other authors Salimov, A. A., A $\breve{g c a}$, F. [2, 10], Yano, K., Ishihara, S. [14], Ocak, F. [8], Gezer, A., Altunbas, M. [5] etc...

The main idea in this note consists in the modification of the Sasaki metric [10]. Firstly we introduce the Berger type deformed Sasaki metric on the cotangent bundle $T^{*} M$ over an anti-paraKähler manifold $(M, \varphi, g)$ and we investigate the Levi-Civita connection of this metric (Theorem 3.1). Secondly we study the harmonicity on cotangent bundle equipped with the Berger type deformed Sasaki metric, then we establish necessary and sufficient conditions under which a covector field is harmonic with respect to the Berger type deformed Sasaki metric (Theorem 4.2, Corollary 4.1 and Theorem 4.3). Next we also construct some examples of harmonic covector fields. Finally we study the harmonicity of the map $\sigma:(M, g) \longrightarrow\left(T^{*} N, \tilde{h}\right)$ (Theorem 4.5 and Theorem 4.6) and the map $\Phi:\left(T^{*} M, \tilde{g}\right) \longrightarrow(N, h)$ (Theorem 4.7 and Theorem 4.8).

Consider a smooth map $\phi:\left(M^{m}, g\right) \rightarrow\left(N^{n}, h\right)$ between two Riemannian manifolds, then the second fundamental form of $\phi$ is defined by

$$
(\nabla d \phi)(X, Y)=\nabla_{X}^{\phi} d \phi(Y)-d \phi\left(\nabla_{X} Y\right)
$$

Received : 11-September-2020, Accepted : 08-October-2020

* Corresponding author 
Here $\nabla$ is the Riemannian connection on $M$ and $\nabla^{\phi}$ is the pull-back connection on the pull-back bundle $\phi^{-1} T N$, and

$$
\tau(\phi)=\operatorname{trace}_{g} \nabla d \phi
$$

is the tension field of $\phi$.

The energy functional of $\phi$ is defined by

$$
E(\phi)=\int_{K} e(\phi) d v_{g}
$$

such that $K$ is any compact of $M$, where

$$
e(\phi)=\frac{1}{2} \operatorname{trace}_{g} h(d \phi, d \phi)
$$

is the energy density of $\phi$.

A map is called harmonic if it is a critical point of the energy functional $E$. For any smooth variation $\left\{\phi_{t}\right\}_{t \in I}$ of $\phi$ with $\phi_{0}=\phi$ and $V=\left.\frac{d}{d t} \phi_{t}\right|_{t=0}$, we have

$$
\left.\frac{d}{d t} E\left(\phi_{t}\right)\right|_{t=0}=-\int_{K} h(\tau(\phi), V) d v_{g}
$$

Then $\phi$ is harmonic if and only if $\tau(\phi)=0$.

One can refer to [7], [6] for background on harmonic maps.

\section{Preliminaries}

Let $\left(M^{m}, g\right)$ be an m-dimensional Riemannian manifold, $T^{*} M$ be its cotangent bundle and $\pi: T^{*} M \rightarrow M$ the natural projection. A local chart $\left(U, x^{i}\right)_{i=\overline{1, m}}$ on $M$ induces a local chart $\left(\pi^{-1}(U), x^{i}, x^{\bar{i}}=p_{i}\right)_{i=\overline{1, m}, \bar{i}=m+i}$ on $T^{*} M$, where $p_{i}$ is the component of covector $p$ in each cotangent space $T_{x}^{*} M, x \in U$ with respect to the natural coframe $d x^{i}$. Let $C^{\infty}(M)$ (resp. $C^{\infty}\left(T^{*} M\right)$ ) be the ring of real-valued $C^{\infty}$ functions on $M\left(\right.$ resp. $\left.T^{*} M\right)$ and $\Im_{s}^{r}(M)$ (resp. $\Im_{s}^{r}\left(T^{*} M\right)$ ) be the module over $C^{\infty}(M)$ (resp. $C^{\infty}\left(T^{*} M\right)$ ) of $C^{\infty}$ tensor fields of type $(r, s)$. Denote by $\Gamma_{i j}^{k}$ the Christoffel symbols of $g$ and by $\nabla$ the Levi-Civita connection of $g$.

We have two complementary distributions on $T^{*} M$, the vertical distribution $V T^{*} M=\operatorname{Ker}(d \pi)$ and the horizontal distribution $H T^{*} M$ that define a direct sum decomposition

$$
T T^{*} M=V T^{*} M \oplus H T^{*} M .
$$

Let $X=X^{i} \frac{\partial}{\partial x^{i}}$ and $\omega=\omega_{i} d x^{i}$ be a local expressions in $\left(U, x^{i}\right)_{i=\overline{1, m}}, U \subset M$ of a vector and covector (1-form) field $X \in \Im_{0}^{1}(M)$ and $\omega \in \Im_{1}^{0}(M)$, respectively. Then the complete and horizontal lifts ${ }^{C} X,{ }^{H} X \in \Im_{0}^{1}\left(T^{*} M\right)$ of $X \in \Im_{0}^{1}(M)$ and the vertical lift ${ }^{V} \omega \in \Im_{0}^{1}\left(T^{*} M\right)$ of $\omega \in \Im_{1}^{0}(M)$ are defined, respectively by

$$
\begin{aligned}
{ }^{C} X & =X^{i} \frac{\partial}{\partial x^{i}}-p_{h} \frac{\partial X^{h}}{\partial x^{i}} \frac{\partial}{\partial x^{\bar{i}}}, \\
{ }^{H} X & =X^{i} \frac{\partial}{\partial x^{i}}+p_{h} \Gamma_{i j}^{h} X^{j} \frac{\partial}{\partial x^{\bar{i}}}, \\
V_{\omega} & =\omega_{i} \frac{\partial}{\partial x^{\bar{i}}},
\end{aligned}
$$

with respect to the natural frame $\left\{\frac{\partial}{\partial x^{i}}, \frac{\partial}{\partial x^{i}}\right\}$, (see [14] for more details).

From (2.3) and (2.4) we see that ${ }^{H}\left(\frac{\partial}{\partial x^{i}}\right)$ and ${ }^{V}\left(d x^{i}\right)$ have respectively local expressions of the form

$$
\begin{aligned}
& \tilde{e}_{i}={ }^{H}\left(\frac{\partial}{\partial x^{i}}\right)=\frac{\partial}{\partial x^{i}}+p_{a} \Gamma_{h i}^{a} \frac{\partial}{\partial x^{\bar{h}}}, \\
& \tilde{e}_{\bar{i}}=V_{\left(d x^{i}\right)}=\frac{\partial}{\partial x^{\bar{i}}} .
\end{aligned}
$$


The set of vector fields $\left\{\tilde{e}_{i}\right\}$ on $\pi^{-1}(U)$ define a local frame for $H T^{*} M$ over $\pi^{-1}(U)$ and the set of vector fields $\left\{\tilde{e}_{\bar{i}}\right\}$ on $\pi^{-1}(U)$ define a local frame for $V T^{*} M$ over $\pi^{-1}(U)$. The set $\left\{\tilde{e}_{\alpha}\right\}=\left\{\tilde{e}_{i}, \tilde{e}_{\bar{i}}\right\}$ define a local frame on $T^{*} M$, adapted to the direct sum decomposition (2.1). The indices $\alpha, \beta, \ldots=\overline{1,2 m}$ indicate the indices with respect to the adapted frame .

Using (2.3), (2.4) we have,

$$
\begin{aligned}
& { }^{H} X=X^{i} \tilde{e}_{i}, \quad{ }^{H} X=\left(\begin{array}{c}
X^{i} \\
0
\end{array}\right), \\
& V_{\omega}=\omega_{i} \tilde{e}_{\bar{i}}, \quad V_{\omega}=\left(\begin{array}{c}
0 \\
\omega_{i}
\end{array}\right),
\end{aligned}
$$

with respect to the adapted frame $\left\{\tilde{e}_{\alpha}\right\}_{\alpha=\overline{1,2 m}}$, (see [14] for more details).

Lemma 2.1. [14] Let $\left(M^{m}, g\right)$ be a Riemannian manifold. The bracket operation of vertical and horizontal vector fields on $T^{*} M$ is given by the formulas

(1) $\left[{ }^{V} \omega,{ }^{V} \theta\right]=0$,

(2) $\left[{ }^{H} X,{ }^{V} \theta\right]={ }^{V}\left(\nabla_{X} \theta\right)$,

(3) $\left[{ }^{H} X,{ }^{H} Y\right]={ }^{H}[X, Y]+{ }^{V}(p R(X, Y))$,

for all vector fields $X, Y \in \Im_{0}^{1}(M)$ and $\omega, \theta \in \Im_{1}^{0}(M)$, where $\nabla$ and $R$ denotes respectively the Levi-Civita connection and the curvature tensor of $\left(M^{m}, g\right)$.

Let $\left(M^{m}, g\right)$ be a Riemannian manifold, we define to maps

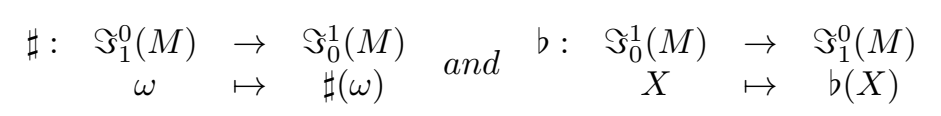

by $g(\sharp(\omega), Y)=\omega(Y)$ and $b(X)(Y)=g(X, Y)$ respectively for all $Y \in \Im_{0}^{1}(M)$.

Locally for all $X=X^{i} \frac{\partial}{\partial x^{i}} \in \Im_{0}^{1}(M)$ and $\omega=\omega_{i} d x^{i} \in \Im_{1}^{0}(M)$, we have

$$
\sharp(\omega)=g^{i j} \omega_{i} \frac{\partial}{\partial x^{j}} \text { and } b(X)=g_{i j} X^{i} d x^{j}
$$

where $\left(g^{i j}\right)$ is the inverse matrix of the matrix $\left(g_{i j}\right)$.

For each $x \in M$ the scalar product $g^{-1}=\left(g^{i j}\right)$ is defined on the cotangent space $T_{x}^{*} M$ by, for all $\omega, \theta \in \Im_{1}^{0}(M)$

$$
g^{-1}(\omega, \theta)=g(\sharp(\omega), \sharp(\theta))=g^{i j} \omega_{i} \theta_{j} .
$$

In this case we have $\sharp(\omega)=g^{-1} \circ \omega$ and $b(X)=g \circ X$.

In the following, we noted $\sharp(\omega)$ by $\tilde{\omega}$ and $b(X)$ by $\tilde{X}$ for all $X \in \Im_{0}^{1}(M)$ and $\omega \in \Im_{1}^{0}(M)$.

Lemma 2.2. Let $(M, g)$ be a Riemannian manifold, we have the following.

$$
\begin{aligned}
\nabla_{X} \tilde{\omega} & =\widetilde{\nabla_{X} \omega}, \\
X g^{-1}(\omega, \theta) & =g^{-1}\left(\nabla_{X} \omega, \theta\right)+g^{-1}\left(\omega, \nabla_{X} \theta\right),
\end{aligned}
$$

for all $X \in \Im_{0}^{1}(M), \omega, \theta \in \Im_{1}^{0}(M)$ and $\varphi \in \Im_{1}^{1}(M)$, where $\nabla$ is the Levi-Civita connection of $(M, g)$.

Proof. For all $Y \in \Im_{0}^{1}(M)$,

$$
\begin{aligned}
g\left(\nabla_{X} \tilde{\omega}, Y\right) & =X(g(\tilde{\omega}, Y))-g\left(\tilde{\omega}, \nabla_{X} Y\right) \\
& =X(\omega(Y))-\omega\left(\nabla_{X} Y\right) \\
& =\left(\nabla_{X} \omega\right)(Y) \\
& =g\left(\widetilde{\nabla_{X} \omega}, Y\right), \\
X g^{-1}(\omega, \theta)= & X g(\tilde{\omega}, \tilde{\theta}) \\
= & g\left(\nabla_{X} \tilde{\omega}, \tilde{\theta}\right)+g\left(\tilde{\omega}, \nabla_{X} \tilde{\theta}\right) \\
= & g\left(\widetilde{\nabla_{X} \omega}, \tilde{\theta}\right)+g\left(\tilde{\omega}, \widetilde{\nabla_{X} \theta}\right) \\
= & g^{-1}\left(\nabla_{X} \omega, \theta\right)+g^{-1}\left(\omega, \nabla_{X} \theta\right) .
\end{aligned}
$$




\section{Berger type deformed Sasaki metric}

Let $M$ be a $2 m$-dimensional Riemannian manifold with a Riemannian metric $g$. An almost paracomplex manifold is an almost product manifold $\left(M^{2 m}, \varphi\right), \varphi^{2}=i d$, such that the two eigenbundles $T^{+} M$ and $T^{-} M$ associated to the two eigenvalues +1 and -1 of $\varphi$, respectively, have the same rank.

The integrability of an almost paracomplex structure is equivalent to the vanishing of the Nijenhuis tensor:

$$
N_{\varphi}(X, Y)=[\varphi X, \varphi Y]-\varphi[\varphi X, Y]-\varphi[X, \varphi Y]+[X, Y] .
$$

A paracomplex structure is an integrable almost paracomplex structure.

Let $\left(M^{2 m}, \varphi\right)$ be an almost paracomplex manifold. A Riemannian metric $g$ is said to be an anti-paraHermitian metric (B-metric)[11] if

$$
g(\varphi X, \varphi Y)=g(X, Y) \Leftrightarrow g(\varphi X, Y)=g(X, \varphi Y)
$$

for all $X, Y \in \Im_{0}^{1}(M)$

If $\left(M^{2 m}, \varphi\right)$ is an almost paracomplex manifold with an anti-paraHermitian metric $g$, then the triple $\left(M^{2 m}, \varphi, g\right)$ is said to be an almost anti-paraHermitian manifold (an almost B-manifold)[11]. Moreover, $\left(M^{2 m}, \varphi, g\right)$ is said to be anti-paraKä manifold (B-manifold)[11] if $\varphi$ is parallel with respect to the Levi-Civita connection $\nabla$ of $g$ i.e $(\nabla \varphi=0)$.

It is well known that if $\left(M^{2 m}, \varphi, g\right)$ is a anti-paraKähler manifold, the Riemannian curvature tensor is pure [11], and for all $Y, Z \in \Im_{0}^{1}(M)$.

$$
\begin{cases}R(\varphi Y, Z) & =R(Y, \varphi Z)=R(Y, Z) \varphi=\varphi R(Y, Z) \\ R(\varphi Y, \varphi Z) & =R(Y, Z)\end{cases}
$$

Definition 3.1. Let $\left(M^{2 m}, \varphi, g\right)$ be an almost anti-paraHermitian manifold and $T^{*} M$ be its tangent bundle. A fiber-wise Berger type deformation of the Sasaki metric noted $\tilde{g}$ is defined on $T^{*} M$ by

$$
\begin{aligned}
\tilde{g}\left({ }^{H} X,{ }^{H} Y\right) & =g(X, Y)=g(X, Y) \circ \pi, \\
\tilde{g}\left({ }^{H} X,{ }^{V} \theta\right) & =0, \\
\tilde{g}\left({ }^{V} \omega,{ }^{V} \theta\right) & =g^{-1}(\omega, \theta)+\delta^{2} g^{-1}(\omega, p \varphi) g^{-1}(\theta, p \varphi),
\end{aligned}
$$

for all $X, Y \in \Im_{0}^{1}(M), \omega, \theta \in \Im_{1}^{0}(M)$, where $\delta$ is some constant [3, 13].

Since any tensor field of type $(0, s)$ on $T^{*} M$ where $s \geq 1$ is completely determined with the vector fields of type ${ }^{H} X$ and ${ }^{V} \omega$ where $X \in \Im_{0}^{1}(M)$ and $\omega \in \Im_{1}^{0}(M)$ (see [14]). In the particular case the metric $\tilde{g}$ is tensor field of type $(0,2)$ on $T^{*} M$. It follows that $\tilde{g}$ is completely determined by its formulas (3.3), (3.4) and (3.5).

By means of (2.2) and (2.3), the complete lift ${ }^{C} X$ of $X \in \Im_{0}^{1}(M)$ is given by

$$
{ }^{C} X={ }^{H} X-{ }^{V}(p(\nabla X))
$$

where $p(\nabla X)=(p(\nabla X))_{i} d x^{i}=p_{h}(\nabla X)_{i}^{h} d x^{i}=p_{h}\left(\frac{\partial X^{h}}{\partial x^{i}}+\Gamma_{i j}^{h} X^{j}\right) d x^{i}$.

Taking account of (3.3), (3.4), (3.5) and (3.6), we obtain

$$
\begin{aligned}
\tilde{g}\left({ }^{C} X,{ }^{C} Y\right)= & { }^{V}(g(X, Y))+g^{-1}(p(\nabla X), p(\nabla Y)) \\
& +\delta^{2} g^{-1}(p(\nabla X), p \varphi) g^{-1}(p(\nabla Y), p \varphi) .
\end{aligned}
$$

where

$$
\begin{gathered}
g^{-1}(p(\nabla X), p(\nabla Y))=g^{i j}(p(\nabla X))_{i}(p(\nabla Y))_{j}=g^{i j} p_{h} p_{k}(\nabla X)_{i}^{h}(\nabla Y)_{j}^{k}, \\
g^{-1}(p(\nabla X), p \varphi)=g^{i j}(p(\nabla X))_{i}(p \varphi)_{j}=g^{i j} p_{h} p_{k}(\nabla X)_{i}^{h} \varphi_{j}^{k} .
\end{gathered}
$$

Since the tensor field $\tilde{g} \in \Im_{2}^{0}\left(T^{*} M\right)$ is completely determined also by its action on vector fields of type ${ }^{C} X$ and ${ }^{C} Y$ (see [14]), we say that formula (3.7) is an alternative characterization of $\tilde{g}$.

Remark 3.1. From formulas (3.3), (3.4), (3.5), the Berger type deformed Sasaki metric $\tilde{g}$ has components

$$
\tilde{g}=\left(\begin{array}{cc}
g_{i j} & 0 \\
0 & g^{i j}+\delta^{2} g^{i t} g^{j s}(p \varphi)_{t}(p \varphi)_{s}
\end{array}\right)
$$

with respect to the adapted frame $\left\{\tilde{e}_{\alpha}\right\}_{\alpha=\overline{1,2 m}}$. 
Lemma 3.1. Let $\left(M^{2 m}, \varphi, g\right)$ be an anti-paraKähler manifold, we have the following:

1. ${ }^{H} X g^{-1}(\theta, \eta)=X g^{-1}(\theta, \eta)=g^{-1}\left(\nabla_{X} \theta, \eta\right)+g^{-1}\left(\theta, \nabla_{X} \eta\right)$,

2. ${ }^{H} X g^{-1}(\theta, p \varphi)=g^{-1}\left(\nabla_{X} \theta, p \varphi\right)$,

3. $V_{\omega g^{-1}}(\theta, p \varphi)=g^{-1}(\theta, \omega \varphi)$,

4. $V_{\omega g}-1(\theta, \eta)=0$,

for all $X \in \Im_{0}^{1}(M)$ and $\omega, \theta, \eta \in \Im_{1}^{0}(M)$.

Lemma 3.2. Let $\left(M^{2 m}, \varphi, g\right)$ be an anti-paraKähler manifold and $\left(T^{*} M, \tilde{g}\right)$ its cotangent bundle equipped with the Berger type deformed Sasaki metric, we have the following:

$$
\begin{aligned}
& { }^{{ }^{H}} X \tilde{g}\left({ }^{V} \theta,{ }^{V} \eta\right)=\tilde{g}\left({ }^{V}\left(\nabla_{X} \theta\right),{ }^{V} \eta\right)+\tilde{g}\left({ }^{V} \theta,{ }^{V}\left(\nabla_{X} \eta\right)\right) \\
& \text { (2) }{ }^{V_{\omega}} \tilde{g}\left({ }^{V} \theta,{ }^{V} \eta\right)
\end{aligned}
$$

for all $X \in \Im_{0}^{1}(M)$ and $\omega, \theta, \eta \in \Im_{1}^{0}(M)$.

Proof. From Lemma 3.1, we have

$$
\text { (1) } \begin{aligned}
{ }^{H} X \tilde{g}\left({ }^{V} \theta,{ }^{V} \eta\right) & ={ }^{H} X\left[g^{-1}(\theta, \eta)+\delta^{2} g^{-1}(\theta, p \varphi) g^{-1}(\eta, p \varphi)\right] \\
& \left.={ }^{H} X\left(g^{-1}(\theta, \eta)\right)+\delta^{2 H} X\left(g^{-1}(\theta, p \varphi)\right) g^{-1}(\eta, p \varphi)\right)+\delta^{2} g^{-1}(\theta, p \varphi)^{H} X\left(g^{-1}(\eta, p \varphi)\right) \\
& =g^{-1}\left(\nabla_{X} \theta, \eta\right)+g^{-1}\left(\theta, \nabla_{X} \eta\right)+\delta^{2} g^{-1}\left(\nabla_{X} \theta, p \varphi\right) g^{-1}(\eta, p \varphi)+\delta^{2} g^{-1}(\theta, p \varphi) g^{-1}\left(\nabla_{X} \eta, p \varphi\right) \\
& =\tilde{g}\left({ }^{V}\left(\nabla_{X} \theta\right),{ }^{V} \eta\right)+\tilde{g}\left({ }^{V} \theta,{ }^{V}\left(\nabla_{X} \eta\right)\right) . \\
(2) V_{\omega} \tilde{g}\left({ }^{V} \theta,{ }^{V} \eta\right) & =V_{\omega}\left[g^{-1}(\theta, \eta)+\delta^{2} g^{-1}(\theta, p \varphi) g^{-1}(\eta, p \varphi)\right] \\
& =V_{\omega}\left(g^{-1}(\theta, \eta)\right)+\delta^{2} V_{\omega}\left(g^{-1}(\theta, p \varphi)\right) g^{-1}(\eta, p \varphi)+\delta^{2} g^{-1}(\theta, p \varphi)^{V} \omega\left(g^{-1}(\eta, p \varphi)\right) \\
& =\delta^{2} g^{-1}(\theta, \omega \varphi) g^{-1}(\eta, p \varphi)+\delta^{2} g^{-1}(\theta, p \varphi) g^{-1}(\eta, \omega \varphi) .
\end{aligned}
$$

Theorem 3.1. Let $\left(M^{2 m}, \varphi, g\right)$ be an anti-paraKähler manifold and $\left(T^{*} M, \tilde{g}\right)$ its cotangent bundle equipped with the Berger type deformed Sasaki metric. The Levi-Civita connection $\widetilde{\nabla}$ of the Berger type deformed Sasaki metric $\tilde{g}$ on $T^{*} M$ satisfies the following properties:

$$
\begin{aligned}
& \text { (1) } \widetilde{\nabla}_{{ }^{H}}{ }^{H} Y={ }^{H}\left(\nabla_{X} Y\right)+\frac{1}{2} V(p R(X, Y)) \text {, } \\
& \text { (2) } \left.\widetilde{\nabla}_{{ } X}{ }^{V} \theta=V_{(} \nabla_{X} \theta\right)+\frac{1}{2}{ }^{H}(R(\tilde{p}, \tilde{\theta}) X) \text {, } \\
& \text { (3) } \widetilde{\nabla}_{V_{\omega}}{ }^{H} Y=\frac{1}{2}{ }^{H}(R(\tilde{p}, \tilde{\omega}) Y) \text {, } \\
& \text { (4) } \widetilde{\nabla}_{V_{\omega}} V_{\theta}=\frac{\delta^{2}}{1+\delta^{2} \alpha} g^{-1}(\omega, \theta \varphi)^{V}(p \varphi) \text {, }
\end{aligned}
$$

for all $X, Y \in \Im_{0}^{1}(M), \omega, \theta \in \Im_{1}^{0}(M)$ and $\alpha=g^{-1}(p, p)$, where $\nabla$ and $R$ denotes respectively the Levi-Civita connection and the curvature tensor of $\left(M^{2 m}, \varphi, g\right)$.

The proof of Theorem 3.1 follows directly from Kozul formula and Lemma 3.2 (see [15] for more similar details).

\section{Berger-type deformed Sasaki metric and Harmonicity.}

4.1. Harmonic sections $\omega:\left(M^{2 m}, \varphi, g\right) \longrightarrow\left(T^{*} M, \tilde{g}\right)$

Lemma 4.1. [15] Let $(M, g)$ be a Riemannian manifold. If $\omega \in \Im_{1}^{0}(M)$ is a covector field (1-form) on $M$ and $\xi=(x, p) \in$ $T^{*} M$ such that $\omega_{x}=p$, then we have:

$$
d_{x} \omega\left(X_{x}\right)={ }^{H} X_{\xi}+{ }^{V}\left(\nabla_{X} \omega\right)_{\xi}
$$

where $X \in \Im_{0}^{1}(M)$ and $\nabla$ denote the Levi-Civita connection of $(M, g)$. 
Proof. Let $\left(U, x^{i}\right)$ be a local chart on $M$ in $x \in M$ and $\left(\pi^{-1}(U), x^{i}, p_{i}\right)$ be the induced chart on $T^{*} M$, if $X_{x}=$ $\left.X^{i}(x) \frac{\partial}{\partial x^{i}}\right|_{x}$ and $\omega_{x}=\left.\omega_{i}(x) d x^{i}\right|_{x}=p$, then

$$
\begin{aligned}
d_{x} \omega\left(X_{x}\right) & =\left.X^{i}(x) \frac{\partial}{\partial x^{i}}\right|_{\xi}+\left.X^{i}(x) \frac{\partial \omega_{j}}{\partial x^{i}}(x) \frac{\partial}{\partial p_{j}}\right|_{\xi} \\
& =\left.X^{i}(x) \frac{\partial}{\partial x^{i}}\right|_{\xi}+\left.\omega_{k}(x) \Gamma_{j i}^{k}(x) X^{j}(x) \frac{\partial}{\partial p_{i}}\right|_{\xi}-\left.\omega_{k}(x) \Gamma_{j i}^{k}(x) X^{j}(x) \frac{\partial}{\partial p_{i}}\right|_{\xi}+\left.X^{i}(x) \frac{\partial \omega_{j}}{\partial x^{i}}(x) \frac{\partial}{\partial p_{j}}\right|_{\xi} \\
& =\left.X^{i}(x) \frac{\partial}{\partial x^{i}}\right|_{\xi}+\left.p_{k} \Gamma_{j i}^{k}(x) X^{j}(x) \frac{\partial}{\partial p_{i}}\right|_{\xi}+\left.X^{i}(x) \frac{\partial \omega_{j}}{\partial x^{i}}(x) \frac{\partial}{\partial p_{j}}\right|_{\xi}-\left.\omega_{k}(x) \Gamma_{i j}^{k}(x) X^{i}(x) \frac{\partial}{\partial p_{j}}\right|_{\xi} \\
& ={ }^{H} X_{\xi}+X^{i}(x)\left[\frac{\partial \omega_{j}}{\partial x^{i}}(x)-\omega_{k}(x) \Gamma_{i j}^{k}(x) X^{i}(x)\right]^{V}\left(d x^{i}\right)_{\xi} \\
& ={ }^{H} X_{\xi}+{ }^{V}\left(\nabla_{X} \omega\right)_{\xi} .
\end{aligned}
$$

Lemma 4.2. Let $\left(M^{2 m}, \varphi, g\right)$ be an anti-paraKähler manifold and $\left(T^{*} M, \tilde{g}\right)$ its cotangent bundle equipped with the Berger-type deformed Sasaki metric. If $\omega \in \Im_{1}^{0}(M)$, then the energy density associated to $\omega$ is given by:

$$
e(\omega)=m+\frac{1}{2} \operatorname{trace}_{g}\left[g^{-1}(\nabla \omega, \nabla \omega)+\delta^{2} g^{-1}(\nabla \omega, \omega \varphi)^{2}\right] .
$$

Proof. Let $\xi=(x, p) \in T^{*} M, \omega \in \Im_{1}^{0}(M), \omega_{x}=p$ and $\left(E_{1}, \cdots, E_{2 m}\right)$ be a locale orthonormal frame on $M$, then:

$$
\begin{aligned}
e(\omega)_{x} & =\frac{1}{2} \text { trace }_{g} \tilde{g}(d \omega, d \omega)_{\xi} \\
& =\frac{1}{2} \sum_{i=1}^{2 m} \tilde{g}\left(d \omega\left(E_{i}\right), d \omega\left(E_{i}\right)\right)_{\xi}
\end{aligned}
$$

Using Lemma 4.1, we obtain:

$$
\begin{aligned}
e(\omega) & =\frac{1}{2} \sum_{i=1}^{2 m} \tilde{g}\left({ }^{H} E_{i}+{ }^{V}\left(\nabla_{E_{i}} \omega\right),{ }^{H} E_{i}+{ }^{V}\left(\nabla_{E_{i}} \omega\right)\right) \\
& =\frac{1}{2} \sum_{i=1}^{2 m}\left\{\tilde{g}\left({ }^{H} E_{i},{ }^{H} E_{i}\right)+\tilde{g}\left({ }^{V}\left(\nabla_{E_{i}} \omega\right),{ }^{V}\left(\nabla_{E_{i}} \omega\right)\right)\right\} \\
& =\frac{1}{2} \sum_{i=1}^{2 m}\left\{g\left(E_{i}, E_{i}\right)+g^{-1}\left(\nabla_{E_{i}} \omega, \nabla_{E_{i}} \omega\right)+\delta^{2} g^{-1}\left(\nabla_{E_{i}} \omega, \omega \varphi\right)^{2}\right\} \\
& =m+\frac{1}{2} \text { trace }_{g}\left[g^{-1}(\nabla \omega, \nabla \omega)+\delta^{2} g^{-1}(\nabla \omega, \omega \varphi)^{2}\right] .
\end{aligned}
$$

Theorem 4.1. Let $\left(M^{2 m}, \varphi, g\right)$ be an anti-paraKähler manifold and $\left(T^{*} M, \tilde{g}\right)$ its cotangent bundle equipped with the Berger type deformed Sasaki metric.

If $\omega \in \Im_{1}^{0}(M)$, then the tension field associated to $\omega$ is given by:

$$
\tau(\omega)=H\left[\operatorname{trace}_{g}(R(\tilde{\omega}, \widetilde{\nabla \omega}) *)\right]+V\left[\operatorname{trace}_{g}\left(\nabla^{2} \omega+\frac{\delta^{2}}{1+\delta^{2} \alpha} g^{-1}(\nabla \omega,(\nabla \omega) \varphi) \omega \varphi\right)\right],
$$

where $\alpha=g^{-1}(\omega, \omega)=\|\omega\|^{2}$. 
Proof. Let $(x, p) \in T^{*} M, \omega \in \Im_{1}^{0}(M), \omega_{x}=p$ and $\left\{E_{i}\right\}_{i=1,2 m}$ be a locale orthonormal frame on $M$ such that $\left(\nabla_{E_{i}}^{M} E_{i}\right)_{x}=0$, then

$$
\begin{aligned}
\tau(\omega)_{x} & =\sum_{i=1}^{2 m}\left\{\left(\nabla_{E_{i}}^{\omega} d \omega\left(E_{i}\right)\right)_{x}-d \omega\left(\nabla_{E_{i}}^{M} E_{i}\right)_{x}\right\} \\
& =\sum_{i=1}^{2 m}\left\{\widetilde{\nabla}_{d \omega\left(E_{i}\right)} d \omega\left(E_{i}\right)\right\}_{(x, p)} \\
& \left.=\sum_{i=1}^{2 m}\left\{\widetilde{\nabla}_{\left({ }^{H} E_{i}+V^{V}\left(\nabla_{E_{i}} \omega\right)\right)}\left({ }^{H} E_{i}+V_{\left(\nabla_{E_{i}}\right.} \omega\right)\right)\right\}_{(x, p)} \\
& =\sum_{i=1}^{2 m}\left\{\widetilde{\nabla}_{H_{E_{i}}}{ }^{H} E_{i}+\widetilde{\nabla}_{H_{E_{i}}}{ }^{V}\left(\nabla_{E_{i}} \omega\right)+\widetilde{\nabla}_{V\left(\nabla_{E_{i}} \omega\right)}{ }^{H} E_{i}+\widetilde{\nabla}_{V\left(\nabla_{E_{i}} \omega\right)} V^{V}\left(\nabla_{E_{i}} \omega\right)\right\}_{(x, p)}
\end{aligned}
$$

Using Theorem 3.1, we obtain

$$
\begin{aligned}
\tau(\omega)= & \sum_{i=1}^{2 m}\left[H_{(}\left(\nabla_{E_{i}} E_{i}\right)-\frac{1}{2} V_{(}\left(\omega R\left(E_{i}, E_{i}\right)\right)+{ }^{V}\left(\nabla_{E_{i}} \nabla_{E_{i}} \omega\right)+\frac{1}{2} H\left(R\left(\tilde{\omega}, \widetilde{\nabla_{E_{i}} \omega}\right) E_{i}\right)+\frac{1}{2} H\left(R\left(\tilde{\omega}, \widetilde{\nabla_{E_{i}} \omega}\right) E_{i}\right)\right. \\
& +\frac{\delta^{2}}{1+\delta^{2} \alpha} g^{-1}\left(\nabla_{E_{i}} \omega,\left(\nabla_{E_{i}} \omega\right) \varphi\right)^{V}(\omega \varphi) \\
= & \sum_{i=1}^{2 m}\left[H_{(}\left(R\left(\tilde{\omega}, \widetilde{\nabla_{E_{i}} \omega}\right) E_{i}\right)+{ }^{V}\left(\nabla_{E_{i}} \nabla_{E_{i}} \omega\right)+\frac{\delta^{2}}{1+\delta^{2} \alpha} g^{-1}\left(\nabla_{E_{i}} \omega,\left(\nabla_{E_{i}} \omega\right) \varphi\right)^{V}(\omega \varphi)\right] \\
= & H\left[\operatorname{trace}_{g}(R(\tilde{\omega}, \widetilde{\nabla \omega}) *)\right]+V\left[\text { trace }_{g}\left(\nabla^{2} \omega+\frac{\delta^{2}}{1+\delta^{2} \alpha} g^{-1}(\nabla \omega,(\nabla \omega) \varphi) \omega \varphi\right)\right] .
\end{aligned}
$$

Theorem 4.2. Let $\left(M^{2 m}, \varphi, g\right)$ be an anti-paraKähler manifold and $\left(T^{*} M, \tilde{g}\right)$ its cotangent bundle equipped with the Berger type deformed Sasaki metric. If $\omega \in \Im_{1}^{0}(M), \omega$ is harmonic covector field if and only the following conditions are verified

$$
\text { trace }_{g}(R(\tilde{\omega}, \widetilde{\nabla \omega}) *)=0,
$$

and

$$
\operatorname{trace}_{g}\left(\nabla^{2} \omega+\frac{\delta^{2}}{1+\delta^{2} \alpha} g^{-1}(\nabla \omega,(\nabla \omega) \varphi) \omega \varphi\right)=0,
$$

where $\alpha=g^{-1}(\omega, \omega)=\|\omega\|^{2}$.

Proof. The statement is a direct consequence of Theorem 4.1.

Corollary 4.1. Let $\left(M^{2 m}, \varphi, g\right)$ be an anti-paraKähler manifold and $\left(T^{*} M, \tilde{g}\right)$ its cotangent bundle equipped with the Berger type deformed Sasaki metric.

If $\omega \in \Im_{1}^{0}(M)$, $\omega$ is a parallel covector field (i.e $\nabla \omega=0$ ) then $\omega$ is harmonic.

Example 4.1. Let $\left(\mathbb{R}^{2}, \varphi, g\right)$ be an anti-paraKähler manifold such that

$$
g=e^{2 x} d x^{2}+e^{2 y} d y^{2} .
$$

and

$$
\varphi \frac{\partial}{\partial x}=\frac{e^{x}}{e^{y}} \frac{\partial}{\partial y} \quad, \quad \varphi \frac{\partial}{\partial y}=\frac{e^{y}}{e^{x}} \frac{\partial}{\partial x}
$$

The non-null Christoffel symbols of the Riemannian connection are:

$$
\Gamma_{11}^{1}=\Gamma_{22}^{2}=1
$$


then we have,

$$
\nabla_{\frac{\partial}{\partial x}} d x=-d x, \nabla_{\frac{\partial}{\partial x}} d y=\nabla_{\frac{\partial}{\partial y}} d x=0, \nabla_{\frac{\partial}{\partial y}} d y=-d y,
$$

the covector field $\omega=e^{x} d x+e^{y} d y$ is harmnic because $\omega$ is parallel, indeed,

$$
\begin{aligned}
& \nabla_{\frac{\partial}{\partial x}} \omega=e^{x} d x+e^{x} \nabla_{\frac{\partial}{\partial x}} d x+e^{y} \nabla_{\frac{\partial}{\partial x}} d y=0, \\
& \nabla_{\frac{\partial}{\partial y}} \omega=e^{x} \nabla_{\frac{\partial}{\partial y}} d x+e^{y} d y+e^{y} \nabla_{\frac{\partial}{\partial y}} d y=0,
\end{aligned}
$$

i.e $\nabla \omega=0$, then $\omega$ is harmonic.

Theorem 4.3. Let $\left(M^{2 m}, \varphi, g\right)$ be an anti-paraKähler compact manifold and $\left(T^{*} M, \tilde{g}\right)$ its cotangent bundle equipped with the Berger type deformed Sasaki metric. If $\omega \in \Im_{1}^{0}(M)$, $\omega$ is harmonic covector field if and only if $\omega$ is parallel (i.e $\nabla \omega=0)$.

Proof. If $\omega$ is parallel, from Corollary 4.1, we deduce that $\omega$ is harmonic covector field.

Inversely, let $\phi_{t}$ be a compactly supported variation of $\omega$ defined by:

$$
\begin{aligned}
\mathbb{R} \times M & \longrightarrow T_{x} M \\
(t, x) & \longmapsto \phi_{t}(x)=(1+t) \omega_{x}
\end{aligned}
$$

From lemma 4.2 we have:

$$
\begin{gathered}
e\left(\phi_{t}\right)=m+\frac{(1+t)^{2}}{2} \operatorname{trace}_{g} g^{-1}(\nabla \omega, \nabla \omega)+\frac{(1+t)^{4}}{2} \delta^{2} \text { trace }_{g} g^{-1}(\nabla \omega, \omega \varphi)^{2} \\
E\left(\phi_{t}\right)=m \operatorname{Vol}(M)+\frac{(1+t)^{2}}{2} \int_{M} \operatorname{trace}_{g} g^{-1}(\nabla \omega, \nabla \omega) d v_{g}+\frac{(1+t)^{4}}{2} \delta^{2} \int_{M} \operatorname{trace}_{g} g^{-1}(\nabla \omega, \omega \varphi)^{2} d v_{g}
\end{gathered}
$$

If $\omega$ is a critical point of the energy functional, then we have :

$$
\begin{aligned}
0 & =\left.\frac{\partial}{\partial t} E\left(\phi_{t}\right)\right|_{t=0} \\
& =\frac{\partial}{\partial t}\left[m \operatorname{Vol}(M)+\frac{(1+t)^{2}}{2} \int_{M} \operatorname{trace}_{g} g^{-1}(\nabla \omega, \nabla \omega) d v_{g}\right]_{t=0}+\frac{\partial}{\partial t}\left[\frac{(1+t)^{4}}{2} \delta^{2} \int_{M} \operatorname{trace}_{g} g^{-1}(\nabla \omega, \omega \varphi)^{2} d v_{g}\right]_{t=0} \\
& =\int_{M} \operatorname{trace}_{g} g^{-1}(\nabla \omega, \nabla \omega) d v_{g}+2 \delta^{2} \int_{M} \operatorname{trace}_{g} g^{-1}(\nabla \omega, \omega \varphi)^{2} d v_{g} \\
& =\int_{M} \text { trace }_{g}\left[g^{-1}(\nabla \omega, \nabla \omega)+2 \delta^{2} g^{-1}(\nabla \omega, \omega \varphi)^{2}\right] d v_{g}
\end{aligned}
$$

which gives

$$
g^{-1}(\nabla \omega, \nabla \omega)+2 \delta^{2} g^{-1}(\nabla \omega, \omega \varphi)^{2}=0
$$

hence $\nabla \omega=0$.

Example 4.2. (Counterexample) Let $\left(\mathbb{R}^{2 m}, \varphi,<,>\right)$ be an anti-paraKähler real euclidean space (flat manifold and non compact) and $T^{*} \mathbb{R}^{2 m}$ its cotangent bundle equipped with the Berger type deformed Sasaki metric, such that $\varphi$ is the canonical para complex structure on $\mathbb{R}^{2 m}[4]$ it is given by the matrix

$$
\left(\begin{array}{cc}
0 & I_{m} \\
I_{m} & 0
\end{array}\right)
$$

If $\omega=\left(\omega_{1}, \cdots, \omega_{2 m}\right) \in \Im_{1}^{0}\left(\mathbb{R}^{2 m}\right)$ is a covector field on $\mathbb{R}^{2 m}$

For $\delta=0$, we have

$$
\tau(\omega)=\text { trace }_{g} \nabla^{2} \omega=\left(\sum_{i=1}^{2 m} \frac{\partial^{2} \omega_{1}}{\partial x_{i}^{2}}, \cdots, \sum_{i=1}^{2 m} \frac{\partial^{2} \omega_{2 m}}{\partial x_{i}^{2}}\right),
$$

1) If $\omega$ is constant, then $\omega$ is harmonic.

2) If $\omega_{i}=a_{i} x_{i}$ and $a_{i} \neq 0$, then $\omega$ is harmonic $(\tau(\omega)=0)$ but $\nabla \omega \neq 0$. indeed

$$
\nabla \omega\left(\frac{\partial}{\partial x_{j}}\right)=\nabla_{\frac{\partial}{\partial x_{j}}} \omega=\sum_{i} a_{i} \nabla_{\frac{\partial}{\partial x_{j}}}\left(x_{i} d x_{i}\right)=\sum_{i} \delta_{i}^{j} a_{i} d x_{i}=a_{j} d x_{j} \neq 0
$$


Remark 4.1. In general, using Corollary 4.1 and Theorem 4.3, we can construct many examples for harmonic covector fields.

Theorem 4.4. Let $\left(\mathbb{R}^{2 m}, \varphi,<,>\right)$ be an anti-paraKähler real euclidean space and $T^{*} \mathbb{R}^{2 m}$ its cotangent bundle equipped with the Berger type deformed Sasaki metric. If $\omega=\left(\omega^{1}, \cdots, \omega^{2 m}\right) \in \Im_{1}^{0}\left(\mathbb{R}^{2 m}\right)$, then $\omega$ is harmonic if and only if $\omega$ verifies the following system of equations

$$
\sum_{i=1}^{2 m}\left[\frac{\partial^{2} \omega_{h}}{\partial\left(x^{i}\right)^{2}}+\frac{\delta^{2}}{1+\delta^{2}\|\omega\|^{2}}\left(\sum_{t, k=1}^{2 m} \frac{\partial \omega_{t}}{\partial x^{i}} \frac{\partial \omega_{k}}{\partial x^{i}} \varphi_{t}^{k}\right) \sum_{t=1}^{2 m} \omega_{t} \varphi_{h}^{t}\right]=0 .
$$

for all $h=\overline{1,2 m}$, where $\varphi_{h}^{t}$ denoting the components of $\varphi,\left\{\frac{\partial}{\partial x^{i}}\right\}_{i=1,2 m}$ be a canonical frame on $\mathbb{R}^{2 m}$ and $\|\omega\|^{2}=\langle\omega, \omega\rangle$. Proof. Let $\left\{\frac{\partial}{\partial x^{i}}\right\}_{i=\overline{1,2 m}}$ be a canonical frame on $\mathbb{R}^{2 m}$. Using Theorem 4.2, we have, the equation (4.3) is trivial, then

$$
\begin{aligned}
(4.4) & \Leftrightarrow \quad \operatorname{trace}_{g}\left[\nabla^{2} \omega+\frac{\delta^{2}}{1+\delta^{2}\|\omega\|^{2}} g^{-1}(\nabla \omega,(\nabla \omega) \varphi) \omega \varphi\right]=0 \\
& \Leftrightarrow \sum_{i=1}^{2 m}\left[\nabla_{\frac{\partial}{\partial x^{i}}} \nabla_{\frac{\partial}{\partial x^{i}}} \omega+\frac{\delta^{2}}{1+\delta^{2}\|\omega\|^{2}} g^{-1}\left(\nabla_{\frac{\partial}{\partial x^{i}}} \omega,\left(\nabla_{\frac{\partial}{\partial x^{i}}} \omega\right) \varphi\right) \omega \varphi\right]=0 \\
& \Leftrightarrow \sum_{i=1}^{2 m}\left[\sum_{h=1}^{2 m}\left(\frac{\partial^{2} \omega_{h}}{\partial\left(x^{i}\right)^{2}} d x^{h}\right)+\frac{\delta^{2}}{1+\delta^{2}\|\omega\|^{2}}\left(\sum_{t, k=1}^{2 m} \frac{\partial \omega_{t}}{\partial x^{i}} \frac{\partial \omega_{k}}{\partial x^{i}} \varphi_{t}^{k}\right) \sum_{h, t=1}^{2 m} \omega_{t} \varphi_{h}^{t} d x^{h}\right]=0 \\
& \Leftrightarrow \sum_{i=1}^{2 m}\left[\frac{\partial^{2} \omega_{h}}{\partial\left(x^{i}\right)^{2}}+\frac{\delta^{2}}{1+\delta^{2}\|\omega\|^{2}}\left(\sum_{t, k=1}^{2 m} \frac{\partial \omega_{t}}{\partial x^{i}} \frac{\partial \omega_{k}}{\partial x^{i}} \varphi_{t}^{k}\right) \sum_{t=1}^{2 m} \omega_{t} \varphi_{h}^{t}\right]=0,
\end{aligned}
$$

for all $h=\overline{1,2 m}$, where $\varphi_{t}^{h}$ denoting the components of $\varphi$.

Corollary 4.2. Let $\left(\mathbb{R}^{2 m}, \varphi,<,>\right)$ be an anti-paraKähler real euclidean space and $T^{*} \mathbb{R}^{2 m}$ its cotangent bundle equipped with the Berger type deformed Sasaki metric, such that $\varphi$ is the canonical para complex structure on $\mathbb{R}^{2 m}$ [4].

If $\omega=\left(\omega_{1}, \cdots, \omega_{2 m}\right) \in \Im_{1}^{0}\left(\mathbb{R}^{2 m}\right)$ is a covector field on $\mathbb{R}^{2 m}$, then $\omega$ is harmonic if and only if $\omega$ verifies the following system of equations

$$
\begin{gathered}
\sum_{i=1}^{2 m}\left[\frac{\partial^{2} \omega_{h}}{\partial\left(x^{i}\right)^{2}}+\frac{2 \delta^{2}}{1+\delta^{2}\|\omega\|^{2}}\left(\sum_{j=1}^{2 m} \frac{\partial \omega_{j}}{\partial x^{i}} \frac{\partial \omega_{m+j}}{\partial x^{i}}\right) \omega_{m+h}\right]=0, \quad 1 \leq h \leq m . \\
\sum_{i=1}^{2 m}\left[\frac{\partial^{2} \omega_{h}}{\partial\left(x^{i}\right)^{2}}+\frac{2 \delta^{2}}{1+\delta^{2}\|\omega\|^{2}}\left(\sum_{j=1}^{2 m} \frac{\partial \omega_{j}}{\partial x^{i}} \frac{\partial \omega_{m+j}}{\partial x^{i}}\right) \omega_{h}\right]=0, \quad m+1 \leq h \leq 2 m .
\end{gathered}
$$

where $\left\{\frac{\partial}{\partial x^{i}}\right\}_{i=1,2 m}$ be a canonical frame on $\mathbb{R}^{2 m}$.

Remark 4.2. Using Theorem 4.4 and Corollary 4.2 we can construct many examples of non trivial harmonic covector fields.

Example 4.3. Let $\left(\mathbb{R}^{2 m}, \varphi,<,>\right)$ be an anti-paraKähler real euclidean space and $T^{*} \mathbb{R}^{2 m}$ its cotangent bundle equipped with the Berger type deformed Sasaki metric, such that $\varphi$ is the canonical para complex structure on $\mathbb{R}^{2 m}$.

From Corollary 4.2, we deduce that, $\omega=\left(y\left(x_{1}\right), 0, \cdots, 0\right)$ is a harmonic covector field if and only the function $y$ is solution of differential equation:

$$
y^{\prime \prime}=0
$$

i.e $y(x)=a x_{1}+b, a, b \in \mathbb{R}$.

\subsection{Harmonicity of the map $\sigma:(M, g) \longrightarrow\left(T^{*} N, \tilde{h}\right)$}

Lemma 4.3. Let $\phi:\left(M^{m}, g\right) \rightarrow\left(N^{n}, h\right)$ be a smooth map between Riemannian manifolds and let $\sigma: M \longrightarrow T^{*} N a$ smooth map such that $\phi=\pi_{N} \circ \sigma$ where $\pi_{N}: T^{*} N \rightarrow N$ is the canonical projection, then

$$
d \sigma(X)={ }^{H}(d \phi(X))+{ }^{V}\left(\nabla_{X}^{\phi} \sigma\right),
$$

for all $X \in \Im_{0}^{1}(M)$, where $\nabla^{\phi}$ is the pull-back connection. 
Proof. Let $x \in M$ and $\omega \in \Im_{1}^{0}(N)$ such that $\sigma(x)=\left(\phi(x), \omega_{\phi(x)}\right) \in T^{*} N$ and $\omega_{\phi(x)}=q \in T_{\phi(x)}^{*} N$ i.e $\sigma=\omega \circ \phi$, for all $X \in \Im_{0}^{1}(M)$ from Lemma 4.1, we obtain:

$$
\begin{aligned}
d_{x} \sigma\left(X_{x}\right) & =d_{x}(\omega \circ \phi)\left(X_{x}\right) \\
& =d_{\phi(x)} \omega\left(d_{x} \phi\left(X_{x}\right)\right) \\
& ={ }^{H}(d \phi(X))_{(\phi(x), q)}+{ }^{V}\left(\nabla_{d \phi(X)}^{N} \omega\right)_{(\phi(x), q)} \\
& ={ }^{H}(d \phi(X))_{(\phi(x), q)}+{ }^{V}\left(\nabla_{X}^{\phi} \omega \circ \phi\right)_{(\phi(x), q)} \\
& ={ }^{H}(d \phi(X))_{(\phi(x), q)}+{ }^{V}\left(\nabla_{X}^{\phi} \sigma\right)_{(\phi(x), q)} .
\end{aligned}
$$

Theorem 4.5. Let $\left(M^{m}, g\right)$ be a Riemannian manifold, $\left(N^{2 n}, \varphi, h\right)$ be an anti-paraKähler manifold and let $\left(T^{*} N, \tilde{h}\right)$ the cotangent bundle of $N$ equipped with Berger type deformed Sasaki metric. Let $\phi: M \rightarrow N$ be a smooth map and

$$
\begin{aligned}
\sigma: M & \longrightarrow T^{*} N \\
x & \longmapsto(\phi(x), q)
\end{aligned}
$$

a smooth map such that $\phi=\pi_{N} \circ \sigma$ and $q \in T_{\phi(x)}^{*} N$. The tension field of $\sigma$ is given by

$$
\begin{aligned}
\tau(\sigma)= & H\left(\tau(\phi)+\operatorname{trace}_{g} R^{N}\left(\tilde{\sigma}, \widetilde{\nabla^{\phi} \sigma}\right) d \phi(*)\right) \\
& +{ }^{V}\left(\text { trace }_{g}\left[\left(\nabla^{\phi}\right)^{2} \sigma+\frac{\delta^{2}}{1+\delta^{2}\|\sigma\|^{2}} h^{-1}\left(\nabla^{\phi} \sigma,\left(\nabla^{\phi} \sigma\right) \varphi\right) \sigma \varphi\right]\right),
\end{aligned}
$$

where $\|\sigma\|^{2}=h(\sigma, \sigma)$.

Proof. Let $x \in M$ and $\left\{E_{i}\right\}_{i=1, m}$ be a locale orthonormal frame on $M$ such that $\left(\nabla_{E_{i}}^{M} E_{i}\right)_{x}=0$ and $\sigma(x)=$ $(\phi(x), q), q \in T_{\phi(x)}^{*} N$. Using lemma 4.3, we have

$$
\begin{aligned}
\tau(\sigma)_{x} & =\sum_{i=1}^{m}\left\{\left(\nabla_{E_{i}}^{\sigma} d \sigma\left(E_{i}\right)\right)_{x}-d \sigma\left(\nabla_{E_{i}}^{M} E_{i}\right)_{x}\right\} \\
& =\sum_{i=1}^{m}\left\{\nabla_{d \sigma\left(E_{i}\right)}^{T^{*} N} d \sigma\left(E_{i}\right)\right\}_{(\phi(x), q)}
\end{aligned}
$$

Using Lemma 4.3, we obtain:

$$
\begin{aligned}
& \tau(\sigma)_{x}=\sum_{i=1}^{m}\left\{\nabla_{\left({ }^{H}\left(d \phi\left(E_{i}\right)\right)+{ }^{V}\left(\nabla_{E_{i}}^{\phi} \sigma\right)\right)}^{T^{*} N}\left({ }^{H}\left(d \phi\left(E_{i}\right)\right)+{ }^{V}\left(\nabla_{E_{i}}^{\phi} \sigma\right)\right)\right\}_{(\phi(x), q)} \\
& \left.\left.=\sum_{i=1}^{m}\left\{\nabla_{H_{\left(d \phi\left(E_{i}\right)\right)}^{T^{*} N}}^{H}\left(d \phi\left(E_{i}\right)\right)+\nabla_{H\left(d \phi\left(E_{i}\right)\right)}^{T^{*} N} V_{\left(\nabla_{E_{i}}\right.}^{\phi} \sigma\right)+\nabla_{V_{\left(\nabla_{E_{i}}\right.} T^{*} N}^{T^{*}}{ }^{H}\left(d \phi\left(E_{i}\right)\right)+\nabla_{\left.V_{\left(\nabla_{E_{i}}\right.} \sigma\right)}^{T^{*} N} V_{\left(\nabla_{E_{i}}\right.}^{\phi} \sigma\right)\right\}_{(\phi(x), q)}
\end{aligned}
$$

From the theorem 3.1, we obtain:

$$
\begin{aligned}
\tau(\sigma)= & \sum_{i=1}^{m}\left[{ }^{H}\left(\nabla_{d \phi\left(E_{i}\right)}^{N} d \phi\left(E_{i}\right)\right)+\frac{1}{2} V\left(\sigma R^{N}\left(d \phi\left(E_{i}\right), d \phi\left(E_{i}\right)\right)\right)+\frac{1}{2} H^{N}\left(R^{N}\left(\tilde{\sigma}, \widetilde{\nabla^{\phi} \sigma}\right) d \phi\left(E_{i}\right)\right)\right. \\
& \left.+{ }^{V}\left(\nabla_{d \phi\left(E_{i}\right)}^{N} \nabla_{E_{i}}^{\phi} \sigma\right)+\frac{1}{2}{ }^{H}\left(R^{N}\left(\tilde{\sigma}, \widetilde{\nabla^{\phi} \sigma}\right) d \phi\left(E_{i}\right)\right)+\frac{\delta^{2}}{1+\delta^{2}\|\sigma\|^{2}} h^{-1}\left(\nabla_{E_{i}}^{\phi} \sigma,\left(\nabla_{E_{i}}^{\phi} \sigma\right) \varphi\right)^{V}(\sigma \varphi)\right] \\
= & \sum_{i=1}^{m}\left[{ }^{H}\left(\nabla_{E_{i}}^{\phi} d \phi\left(E_{i}\right)\right)+{ }^{H}\left(R^{N}\left(\tilde{\sigma}, \widetilde{\nabla^{\phi} \sigma}\right) d \phi\left(E_{i}\right)\right)+{ }^{V}\left(\nabla_{E_{i}}^{\phi} \nabla_{E_{i}}^{\phi} \sigma\right)+\frac{\delta^{2}}{1+\delta^{2}\|\sigma\|^{2}} h^{-1}\left(\nabla_{E_{i}}^{\phi} \sigma,\left(\nabla_{E_{i}}^{\phi} \sigma\right) \varphi\right)^{V}(\sigma \varphi)\right]
\end{aligned}
$$

Therefore we get

$$
\tau(\sigma)={ }^{H}\left(\tau(\phi)+\operatorname{trace}_{g} R^{N}\left(\tilde{\sigma}, \widetilde{\nabla^{\phi} \sigma}\right) d \phi(*)\right)+{ }^{V}\left(\operatorname{trace}_{g}\left[\left(\nabla^{\phi}\right)^{2} \sigma+\frac{\delta^{2}}{1+\delta^{2}\|\sigma\|^{2}} h^{-1}\left(\nabla^{\phi} \sigma,\left(\nabla^{\phi} \sigma\right) \varphi\right) \sigma \varphi\right]\right) .
$$


From Theorem 4.5 we obtain.

Theorem 4.6. Let $\left(M^{m}, g\right)$ be a Riemannian manifold, $\left(N^{2 n}, \varphi, h\right)$ be an anti-paraKähler manifold and let $\left(T^{*} N, \tilde{h}\right)$ the cotangent bundle of $N$ equipped with Berger type deformed Sasaki metric. Let $\phi: M \rightarrow N$ be a smooth map and

$$
\begin{aligned}
\sigma: M & \longrightarrow T^{*} N \\
x & \longmapsto(\phi(x), q)
\end{aligned}
$$

a smooth map such that $\phi=\pi_{N} \circ \sigma$ and $v \in T_{\phi(x)}^{*} N$, then $\sigma$ is a harmonic if and only if the following conditions are verified

$$
\tau(\phi)=-\operatorname{trace}_{g} R^{N}\left(\tilde{\sigma}, \widetilde{\nabla^{\phi}}\right) d \phi(*)
$$

and

$$
\text { trace }_{g}\left[\left(\nabla^{\phi}\right)^{2} \sigma+\frac{\delta^{2}}{1+\delta^{2}\|\sigma\|^{2}} h^{-1}\left(\nabla^{\phi} \sigma,\left(\nabla^{\phi} \sigma\right) \varphi\right) \sigma \varphi\right]=0
$$

4.3. Harmonicity of the map $\Phi:\left(T^{*} M, \tilde{g}\right) \longrightarrow(N, h)$

Lemma 4.4. Let $\left(M^{2 m}, \varphi, g\right)$ be an anti-paraKähler manifold and $\left(T^{*} M, \tilde{g}\right)$ its cotangent bundle equipped with the Berger type deformed Sasaki metric. The canonical projection

$$
\begin{aligned}
\pi:\left(T^{*} M, \tilde{g}\right) & \longrightarrow(M, g) \\
(x, p) & \longmapsto x
\end{aligned}
$$

is harmonic i.e $\tau(\pi)=0$.

Proof. We put $r=2 m$, Let $\left\{E_{i}\right\}_{i=\overline{1, r}}$ and $\left\{\omega^{i}\right\}_{i=\overline{1, r}}$ be a local orthonormal frame, coframe on $M$ respectively and $\left\{F_{j}\right\}_{j=\overline{1,2 r}}$ be a locale frame on $T^{*} M$ defined by

$$
F_{j}=\left\{\begin{array}{cc}
{ }^{H} E_{j}, & 1 \leq j \leq r \\
V_{\omega^{j-r}}, & r+1 \leq j \leq 2 r
\end{array}\right.
$$

The tension field of $\pi$ is given by

$$
\begin{aligned}
\tau(\pi) & =\operatorname{trace}_{\tilde{g}} \nabla d \pi \\
& =\sum_{i, j=1}^{2 r} \tilde{g}^{i j}\left\{\nabla_{d \pi\left(F_{i}\right)}^{M} d \pi\left(F_{j}\right)-d \pi\left(\nabla_{F_{i}}^{T^{*} M} F_{j}\right)\right\}
\end{aligned}
$$

such that $\left(\tilde{g}^{i j}\right)$ is the inverse matrix of the matrix $\left(\tilde{g}_{i j}\right)$ of $\tilde{g}$ where:

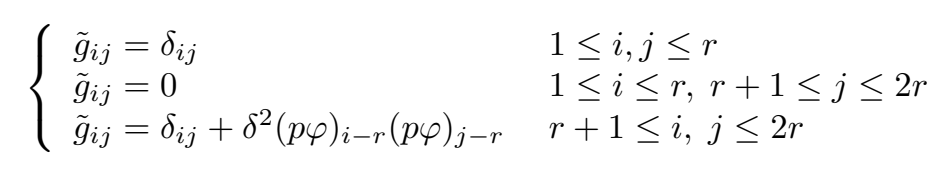

and

$$
\begin{cases}\tilde{g}^{i j}=\delta_{i j} & 1 \leq i, j \leq r \\ \tilde{g}^{i j}=0 & 1 \leq i \leq r, r+1 \leq j \leq 2 r \\ \tilde{g}^{i j}=\frac{\delta_{i j}+\delta^{2}\left(\|p \varphi\|^{2} \delta_{i j}-(p \varphi)_{i-r}(p \varphi)_{j-r}\right)}{1+\delta^{2}\|p \varphi\|^{2}} & r+1 \leq i, j \leq 2 r\end{cases}
$$

where $p \varphi=(p \varphi)_{k} \omega^{k}$, then

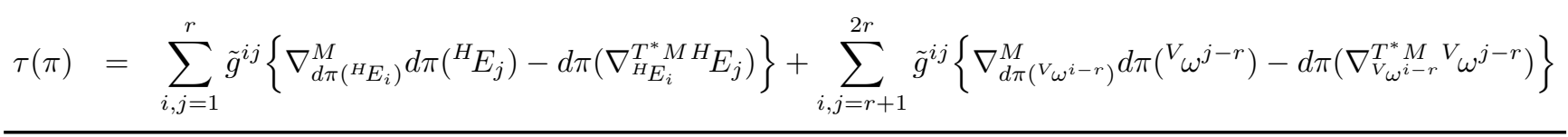


as $d \pi\left({ }^{V} \omega\right)=0$ and $d \pi\left({ }^{H} X\right)=X \circ \pi$ for any $X \in \Im_{0}^{1}(M)$ and $\omega \in \Im_{1}^{0}(M)$ then:

$$
\begin{aligned}
\tau(\pi)= & \sum_{i, j=1}^{r} \tilde{g}^{i j}\left\{\nabla_{\left(E_{i} \circ \pi\right)}^{M}\left(E_{j} \circ \pi\right)-d \pi\left({ }^{H}\left(\nabla_{E_{i}}^{M} E_{j}\right)-\frac{1}{2} V\left(p R\left(E_{i}, E_{j}\right)\right)\right)\right\} \\
& -\sum_{i, j=r+1}^{2 r} \tilde{g}^{i j} \frac{\delta^{2}}{1+\delta^{2} \alpha} g^{-1}\left(\omega^{i-r}, \omega^{j-r} \varphi\right) d \pi\left({ }^{V}(p \varphi)\right) \\
= & \sum_{i, j=1}^{r} \tilde{g}^{i j}\left\{\left(\nabla_{E_{i}}^{M} E_{j}\right) \circ \pi-\left(\nabla_{E_{i}}^{M} E_{j}\right) \circ \pi\right\} \\
= & 0 .
\end{aligned}
$$

Theorem 4.7. Let $\left(M^{2 m}, \varphi, g\right)$ be an anti-paraKähler manifold, $\left(N^{n}, h\right)$ be a Riemannian manifold and let $\left(T^{*} M, \tilde{g}\right)$ the cotangent bundle of $M$ equipped with Berger type deformed Sasaki metric. Let $\phi:(M, g) \longrightarrow(N, h)$ a smooth map. The tension field of the map

$$
\begin{aligned}
\Phi:\left(T^{*} M, \tilde{g}\right) & \longrightarrow(N, h) \\
(x, p) & \longmapsto \phi(x)
\end{aligned}
$$

is given by:

$$
\tau(\Phi)=\tau(\phi) \circ \pi
$$

Proof. We put $r=2 m$, Let $\left\{E_{i}\right\}_{i=\overline{1, r}}$ and $\left\{\omega^{i}\right\}_{i=\overline{1, r}}$ be a local orthonormal frame, coframe on $M$ respectively and $\left\{F_{j}\right\}_{j=1,2 r}$ be a locale frame on $T^{*} M$ defined by (4.13), as the $\Phi$ is defined by $\Phi=\phi \circ \pi$, we have:

$$
\begin{aligned}
\tau(\Phi) & =\tau(\phi \circ \pi) \\
& =d \phi(\tau(\pi))+\operatorname{trace}_{\tilde{g}} \nabla d \phi(d \pi, d \pi) \\
{\text { trace } g^{f}} \nabla d \phi(d \pi, d \pi)= & \sum_{i=1}^{2 r} G^{i j}\left\{\nabla_{d \phi\left(d \pi\left(F_{i}\right)\right)}^{N} d \phi\left(d \pi\left(F_{j}\right)\right)-d \phi\left(\nabla_{d \pi\left(F_{i}\right)}^{M} d \pi\left(F_{j}\right)\right)\right\} \\
= & \sum_{i, j=1}^{r} \delta_{i j}\left[\nabla_{d \phi\left(d \pi\left({ }^{H} E_{i}\right)\right)}^{N} d \phi\left(d \pi\left({ }^{H} E_{j}\right)\right)-d \phi\left(\nabla_{d \pi\left({ }^{H} E_{i}\right)}^{M} d \pi\left({ }^{H} E_{j}\right)\right)\right] \\
= & \sum_{i=1}^{r}\left[\nabla_{d \phi\left(E_{i}\right)}^{N} d \phi\left(E_{i}\right)-d \phi\left(\nabla_{E_{i}}^{M} E_{i}\right)\right] \circ \pi \\
= & \tau(\phi) \circ \pi .
\end{aligned}
$$

Using Lemma 4.4, we obtain:

$$
\tau(\Phi)=\tau(\phi) \circ \pi
$$

Theorem 4.8. Let $\left(M^{2 m}, \varphi, g\right)$ be an anti-paraKähler manifold, $\left(N^{n}, h\right)$ be a Riemannian manifold and let $\left(T^{*} M, \tilde{g}\right)$ the cotangent bundle of $M$ equipped with Berger type deformed Sasaki metric. Let $\phi:(M, g) \longrightarrow(N, h)$ a smooth map. The map

$$
\begin{aligned}
\Phi:\left(T^{*} M, \tilde{g}\right) & \longrightarrow(N, h) \\
(x, p) & \longmapsto \phi(x)
\end{aligned}
$$

is a harmonic if and only if $\phi$ is harmonic. 


\section{Acknowledgments}

The author would like to thank Professor M. Djaa for his guidelines, his helpful comments and suggestions.

\section{References}

[1] Ağca, F.: g-Natural Metrics on the cotangent bundle. Int. Electron. J. Geom. 6(1), 129-146 (2013).

[2] Ağca, F. and Salimov, A. A.: Some notes concerning Cheeger-Gromoll metrics. Hacet. J. Math. Stat. 42(5), 533-549 (2013).

[3] Altunbas, M., Simsek, R., Gezer, A.: A Study Concerning Berger type deformed Sasaki Metric on the Tangent Bundle. Zh. Mat. Fiz. Anal.Geom. 15 (4), 435-447 (2019). https://doi.org/10.15407/mag15.04.435

[4] Cruceanu, V., Fortuny, P., Gadea, P. M.: A survey on paracomplex geometry. Rocky Mountain J. Math. 26 (1), $83-115$ (1996) doi:10.1216/rmjm/1181072105

[5] Gezer, A., Altunbas, M.: On the Rescaled Riemannian Metric of Cheeger Gromoll Type on the Cotangent Bundle. Hacet. J. Math. Stat. 45 (2), 355-365 (2016). https:// Doi:10.15672/HJMS.20164515849

[6] Ells, J., Lemaire, L.: Another report on harmonic maps. Bull. London Math. Soc. 20 (5), 385-524 (1988) https://doi.org/10.1112/blms/20.5.385

[7] Eells, J., Sampson, J. H.: Harmonic mappings of Riemannian manifolds. Amer.J. Math. 86, 109-160 (1964). https://doi.org/10.2307/2373037

[8] Ocak, F. Notes About a New Metric on the Cotangent Bundle, Int. Electron. J. Geom. 12 (2), 241-249 (2019). https://doi.org/10.36890/iejg.542783

[9] Patterson, E. M., Walker, A. G.: Riemannian extensions. Quart. J.Math. Oxford Ser. 2(3), 19-28 (1952).

[10] Salimov, A. A., Agca, F.: Some Properties of Sasakian Metrics in Cotangent Bundles. Mediterr. J. Math. 8(2), $243-255$ (2011). https://doi.org/10.1007/s00009-010-0080-x

[11] Salimov, A. A., Iscan, M., Etayo, F.: Para-holomorphic B-manifold and its properties. Topology Appl. 154(4), 925-933 (2007) https://doi.org/10.1016/j.topol.2006.10.003

[12] Sekizawa, M.: Natural transformations of affine connections on manifolds to metrics on cotangent bundles. In: Proceedings of 14 th Winter School on Abstract Analysis (Srni, 1986), Rend. Circ. Mat. Palermo 14, 129-142 (1987).

[13] Yampolsky, A.: On geodesics of tangent bundle with fiberwise deformed Sasaki metric over Kahlerian manifolds. Zh. Mat. Fiz. Anal. Geom. 8 (2), 177-189 (2012).

[14] Yano, K., Ishihara, S.: Tangent and Cotangent Bundles, M. Dekker, New York, (1973).

[15] Zagane, A.: A new class of metrics on the cotangent bundle. Bull. Transilv. Univ. Brasov Ser. III 13(62)(1), 285-302 (2020). https://doi.org/10.31926/but.mif.2020.13.62.1.22

\section{Affiliations}

ABDERRAHIM ZAGANE

AdDrEss: University Center Ahmed Zabana-Relizane, Dept. of mathematics, 48000, Relizane-Algeria.

E-MAIL: Zaganeabr2018@gmail.com

ORCID ID:0000-0001-9339-3787 